\title{
Role of diffusion-weighted magnetic resonance imaging in assessment of mammographically detected asymmetric densities
}

\author{
Mohamed Zidan ${ }^{1}$, Shimaa Ali Saad ${ }^{1 *}$, Eman Abo Elhamd ${ }^{1}$, Hosam Eldin Galal ${ }^{1}$ and Reem Elkady ${ }^{1,2}$
}

\begin{abstract}
Background: Asymmetric breast density is a potentially perplexing finding; it may be due to normal hormonal variation of the parenchymal pattern and summation artifact or it may indicate an underlying true pathology. The current study aimed to identify the role of diffusion-weighted imaging (DWI) and the apparent diffusion coefficient (ADC) values in the assessment of breast asymmetries.

Results: Fifty breast lesions were detected corresponding to the mammographic asymmetry. There were 35 (70\%) benign lesions and $15(30 \%)$ malignant lesions. The mean ADC value was $1.59 \pm 0.4 \times 10^{-3} \mathrm{~mm}^{2} / \mathrm{s}$ for benign lesions and $0.82 \pm$ $0.3 \times 10^{-3} \mathrm{~mm}^{2} / \mathrm{s}$ for malignant lesions. The ADC cutoff value to differentiate between benign and malignant lesions was $1.10 \times 10^{-3} \mathrm{~mm}^{2} / \mathrm{s}$ with sensitivity $80 \%$, specificity $88.6 \%$, positive predictive value $75 \%$, negative predictive value $91 \%$, and accuracy $86 \%$. Best results were achieved by implementation of the combined DCE-MRI and DWI protocol, with sensitivity 93.3\%, specificity 94.3\%, positive predictive value $87.5 \%$, negative predictive value $97.1 \%$, and accuracy $94 \%$.

Conclusion: Dynamic contrast-enhanced MRI (DCE-MRI) was the most sensitive method for the detection of the underlying malignant pathology of breast asymmetries. However, it provided a limited specificity that may cause improper final BIRADS classification and may increase the unnecessary invasive procedures. DWI was used as an adjunctive method to DCE-MRI that maintained high sensitivity and increased specificity and the overall diagnostic accuracy of breast MRI examination. Best results can be achieved by the combined protocol of DCE-MRI and DWI.
\end{abstract}

Keywords: Diffusion MRl, Dynamic contrast-enhanced MRI, Asymmetry, Focal asymmetry, Global asymmetry, Developing asymmetry

\section{Background}

Asymmetric breast densities are encountered relatively frequently, being reported to occur in $3 \%$ of mammogram. Malignancy can be found in $0-14 \%$ of asymmetric breast tissue biopsies $[1,2]$

Asymmetric breast tissue is usually benign but may indicate a developing mass or an underlying cancer especially if an associated palpable mass is present [3].

\footnotetext{
* Correspondence: drshima15@gmail.com

'Department of Radiology, Assiut University, Asyut, Egypt

Full list of author information is available at the end of the article
}

The American College of Radiology (ACR), Breast Imaging Reporting and Data System (BI-RADS) lexicon, fifth edition provides definitions for four different types of asymmetric breast findings: asymmetry, global asymmetry, focal asymmetry, and developing asymmetry [4].

Asymmetry is an area of fibroglandular density tissue that is visible in only a single view. It should be called an "asymmetry" until it is three-dimensional. It is almost always benign due to summation artifacts (BI-RADS category 1). Routine mammographic screening after 1 year is recommended $[5,6]$. 
Global asymmetry involves a greater volume of breast tissue over a $>1$ quadrant relative to the corresponding region in the contralateral breast. It is often benign and needs no additional evaluation when not associated with palpable abnormalities, architectural distortions, significant calcifications, or masses (BI-RADS category 2) [6].

Focal asymmetry is visible as a confined asymmetry on two views but does not fit the criteria of a mass. It lacks convex outer borders and conspicuity. It involves less than one quadrant of the breast. If no previous mammograms are available for comparison, non-palpable focal asymmetries with no associated abnormalities are often assessed as probably benign (BI-RADS category 3 ), with a recommendation of 6 months follow-up for 1-2 years duration before returning to regular screening. The stability or regression of size for at least 2 years justifies a definitive benign assessment (BI-RADS category 2). Lesion progression effectively establishes the diagnosis of developing asymmetry, which is a focal asymmetry that is new, larger, or denser at the current examination than at previous examinations. It is considered inherently suspicious (BI-RADS category 4) and should be evaluated with additional imaging or biopsy to identify possible cancer $[5,6]$.

Sonomammography may be insufficient to make a final BI-RADS assessment and may have limited sensitivity and specificity for the detection and diagnosis of breast lesions. Moreover, developing asymmetric densities need to be evaluated unless they can be certainly explained in terms of benign causes [7].

In this case, MRI can be used as a supplementary problem-solving procedure. Positive MRI examination will prompt biopsy and allow immediate detection of malignancies that could be missed, while a negative MRI will increase confidence that the indeterminate finding was likely caused by summation artifact or benign tissue and reduce the unnecessary biopsy [8].

Although conventional breast MRI provides (89$100 \%)$ sensitivity in the characterization of breast lesions, there is still an overlap between benign and malignant findings resulting in a variable specificity (50-90\%) [9].

The diffusion-weighted imaging (DWI) provides quantitative and qualitative data reflecting changes at the cellular level and, consequently, unique information on the tumor cellularity and cell membranes integrity. This will increase in breast MRI specificity and reduce unnecessary biopsies [10].

Cell density and extracellular water content affect the Apparent diffusion coefficient values (ADCs) obtained from benign and malignant breast lesions. This allows quantitative assessment of breast lesions with further improvement of MRI specificity [10].

Furthermore, diffusion sequences improve the detection of small lesions, which requires an optimal signalto-noise ratio. It is applicable for small masses and even for non-mass like enhancements which is a common MRI finding in asymmetric breast densities [11].

The aim of this study was to assess the role of diffusion MRI and the ADC value calculation in the assessment of asymmetric densities identified on mammograms.

\section{Methods \\ Study participants}

This study was an analytic prospective study. The study had been approved by our institutional board of ethics with ClinicalTrail.gov registration number (NCT03696147). A written informed consent was obtained from each patient.

The study was conducted in the breast imaging unit during the period from April 2016 to January 2019.

Ninety cases (age range, 22 to 74 years) with asymmetric densities detected on mammography were initially enrolled in this study. These cases were subjected to meticulous examination with complementary views and ultrasound examination side by side with short-term follow-up. Fourteen cases with one view asymmetry (asymmetry) were finally assigned as BIRADS I lesions and were attributed to summation artifacts, cyclic hormonal changes, or normal variations in the glandular parenchyma. Twenty-six cases were focal asymmetry; further assessment with ultrasound revealed asymmetry due to different benign pathologies (BIRADS II lesions). Short-term follow-up confirmed the same findings. The previously mentioned forty patients did not need a further assessment with breast MRI and thus were excluded from the statistical analysis.

Fifty female patients who did not fulfill all the benign criteria of BIRADS lexicon descriptors underwent conventional and diffusion-weighted MRI examination and were included in the study statistical analysis. Imaging findings were correlated with the results of a histopathological evaluation that was done in all cases and was considered as the gold standard management.

\section{Imaging technique Mammography}

Bilateral mammographic examinations in cranio-caudal and medio-lateral oblique projections were performed using (GE Alpha RT). According to BIRADS 5th lexicon, mammograms were evaluated for breast composition, asymmetric density category, and its location; associated calcifications; architectural distortion; associated suspicious features such as nipple retraction, skin thickening, and axillary lymphadenopathy; and assessment of any change from previous examinations when available.

\section{Ultrasound examination}

All patients were subjected to sonographic examination (Philips Affiniti 50). In this study, ultrasound had a crucial role in the determination of the study group 
participants. It provided an overview of the underlying pathology of asymmetry, allowed more precise BIRADS classification, and permitted confident decision for subsequent management especially for cases of one view asymmetry.

The whole breast and axilla were examined thoroughly with special emphasis on the expected site of mammographic detected asymmetry. Color and power Doppler techniques were performed. Second look ultrasound was carried out after MRI examination for masses that were detected only with MRI and were not initially seen by ultrasound. It provided a second chance to evaluate overlooked lesions and schedule it for ultrasound-guided biopsy.

\section{MRI imaging protocol}

All MRI examinations were performed using $1.5 \mathrm{~T}$ systems (Philips Achieva) and (Simens Magnetom Sempra), and four-channel phased array breast coil was used. For all patients, the following sequences were obtained:

- Localizer; axial fast spoiled gradient echo.

- Axial T1 WI (TR/TE, 540/10 ms, field of view $400 \mathrm{~mm}$, slice thickness $3 \mathrm{~mm}$, matrix $340 \times 512$ ).

- Axial T2 WI (TR/TE, 4500/120 ms, field of view $380 \mathrm{~mm}$, slice thickness $3 \mathrm{~mm}$, matrix $340 \times 512$ ).

- Axial T2 fat suppression (TR/TE, 3600/80 ms, field of view $380 \mathrm{~mm}$, slice thickness $3 \mathrm{~mm}$, matrix $340 \times 512$ ).

- Axial EPI-DW (TR/TE, $1500 / 80 \mathrm{~ms}$, field of view $460 \mathrm{~mm}$, slice thickness $4.5 \mathrm{~mm}$, matrix $340 \times 512$ ). DWI was performed prior to the contrast-enhanced examination to avoid the effect of the contrast on the T1 relaxation of the breast tissues. Sensitizing diffusion gradients were applied along the $x, y$, and $z$ directions with different selected $b$ values ( $b$ values of 0, 200, 400, 800, and $1000 \mathrm{~s} / \mathrm{mm} 2$ ) and all ADC values were then demonstrated on an ADC map.

- Axial three-dimensional (3D) dynamic fatsuppressed THRIVE (T1 High-Resolution Isotropic Volumetric Excitation): (TR/TE, 470/10 ms, slice thickness $1 \mathrm{~mm}$, field of view $360 \mathrm{~mm}$, flip angle 90). Intravenous injection of contrast agent, Gadolinium dimeglumine (Magnevist) at a dose of $(0.1 \mathrm{mmol} / \mathrm{kg})$ at a rate of $2 \mathrm{ml} / \mathrm{s}$, followed by a 20 $\mathrm{ml}$ saline flush administered and followed by multiphasic (5 phases) dynamic post-contrast sequences each lasting for about $1 \mathrm{~min}$.

\section{Data processing and image analysis}

Image post-processing techniques were applied for every breast MRI examination. Subtraction images were obtained. ROI measurement of suspicious lesions for parametric color maps to assess the lesion enhancement. Time-intensity curves were obtained.
Axial T1WI, axial T2WI, and T2 fat suppression were examined to assess morphology of breast lesion then subtraction and post-contrast fat-suppressed images were examined to assess lesion enhancement. Lesions were interpreted and classified according to BIRADS 5th edition including the two major descriptors: morphological assessment and enhancement kinetics were done.

- Diffusion-weighted imaging evaluation, including qualitative assessment. The lesion was restricted if showed a high signal corresponding to an enhancing lesion on contrast-enhanced MRI. Also, a quantitative assessment was performed through the creation of the ADC maps. ADC values were automatically calculated by placing the region of interest (ROI) manually within the lesion at different $b$ values. The mean $A D C$ value within the selected ROI was measured and documented.

\section{Statistical analysis}

The collected data were verified, coded, and analyzed using the Statistical Package for Social Sciences (IBM-SPSS/PC/ VER 21). Descriptive statistics were calculated. Chi-square/ Fisher's exact test was used to compare the difference in distribution of frequencies among different groups. Student's $t$ test was calculated to test the mean differences in continuous variables between groups. ROC curve was depicted to investigate the diagnostic performance of the different diagnostic tools for diagnosis of malignancy, analyzed as area under the curve (AUC), standard error (SE), and $95 \%$ CI. Cohen kappa and validity statistics (sensitivity, specificity, positive and negative predictive value (PPV and $\mathrm{NPV})$ ) were calculated to validate the different tools against histopathological findings. Significant test results were considered when $p$ value was $\leq 0.05$.

\section{Results}

In this study, MRI examination was performed to characterize a total of 50 lesions in 50 female patients. The lesions were detected on screening or diagnostic mammography and interpreted as asymmetric density. The age range in our study was $22-74$ years, mean age of $48 \pm 4.25$. Most of the patients $(50 \%)$ were presented in the age group between 40 and 50 years.

Out of the 50 asymmetric densities, 35 (70\%) lesions were benign while $15(30 \%)$ lesions were malignant. The total numbers of benign and malignant lesions as in final histopathological diagnosis for each type of asymmetry.

Among the 50 asymmetric densities, there were $2(4 \%)$ one view asymmetry, 14 (28\%) global asymmetry, 32 (64\%) focal asymmetry, and 2 (4\%) developing asymmetry. The 2 cases of one view asymmetry were benign, while 10/32 (31.25\%) of focal asymmetries, $4 / 15$ (26.6\%) of global asymmetries, and 1/2 (50\%) of developing asymmetry were malignant. 
The calculated sensitivity and specificity of mammography in the characterization of benign and malignant asymmetries were $47 \%$ and $91.5 \%$, respectively. The positive predictive value was $70 \%$, negative predictive value $80 \%$, accuracy $78 \%$, and area under the curve 0.034 .

MRI examination showed that from 50 lesions, 25 (50\%) masses (16 were benign and 9 malignant) were found, and 25 (50\%) were non-mass-like enhancement (19 were benign and 6 malignant).

Twenty-one lesions showed type I curve (all of them were benign), 14 lesions showed type II curve ( 8 were benign and 6 were malignant), 10 lesions showed type III curve ( 1 was benign and 9 were malignant), and 5 lesions showed no enhancement (all were benign).

The calculated sensitivity and specificity of dynamic contrast-enhanced MRI final BI-RADS assessment in the characterization of benign and malignant lesions were $100 \%$ and $74.3 \%$, respectively. The positive predictive value was $62.5 \%$, negative predictive value $100 \%$, accuracy $82 \%$, and area under the curve 0.871 (Fig. 1).

Regarding the qualitative assessment, 23 (46\%) lesions showed facilitated diffusion with low signal in diffusionweighted images (all of them were benign), and 27 (54\%) lesions showed diffusion restriction with high signal in diffusion-weighted images (12 of them were benign and 15 were malignant).

The mean ADC value was $1.59 \pm 0.4 \times 10^{-3} \mathrm{~mm}^{2} / \mathrm{s}$ for benign lesions and $0.82 \pm 0.3 \times 10^{-} 3 \mathrm{~mm}^{2} / \mathrm{s}$ for malignant lesions.
The majority of benign lesions (31 out of 35 , 88.6\%) had ADC value above the calculated cutoff value of $1.1 \times 10^{-3} \mathrm{~mm}^{2} / \mathrm{s}$, while only 4 out of 35 (11.4\%) had ADC value below the cutoff value (the lesions were breast abscesses). The majority of malignant lesions (12 out of $15,80 \%$ ) had ADC value below the calculated cutoff value of $1.1 \times 10^{-3} \mathrm{~mm}^{2} / \mathrm{s}$, while only 3 out of $15(20 \%)$ had ADC value above the cutoff value (the lesions were atypical ductal hyperplasia and ductal carcinoma in situ).

The ROC (receiver operating characteristics) curve analysis yielded an area under the curve (AUC) was 0.941; the best ADC cutoff value to differentiate between benign and malignant lesions was $1.10 \times 10^{-3} \mathrm{~mm}^{2} / \mathrm{s}$ with sensitivity $80 \%$, specificity $88.6 \%$, positive predictive value $75 \%$, negative predictive value $91 \%$, and accuracy 86\% (Fig. 2).

The calculated sensitivity and specificity of the combined protocol of DCE-MRI and DWI in the characterization of benign and malignant lesions were 93.3\% and $94.3 \%$, respectively. The positive predictive value was $87.5 \%$, negative predictive value $97.1 \%$, accuracy $94 \%$, and area under the curve 0.938 (Fig. 3).

\section{Discussion}

Histopathological diagnosis of 50 asymmetric densities revealed 35 (70\%) benign lesions and 15 (30\%) malignant lesions. This is in agreement with a study done by Sickles, who reported that asymmetric densities are

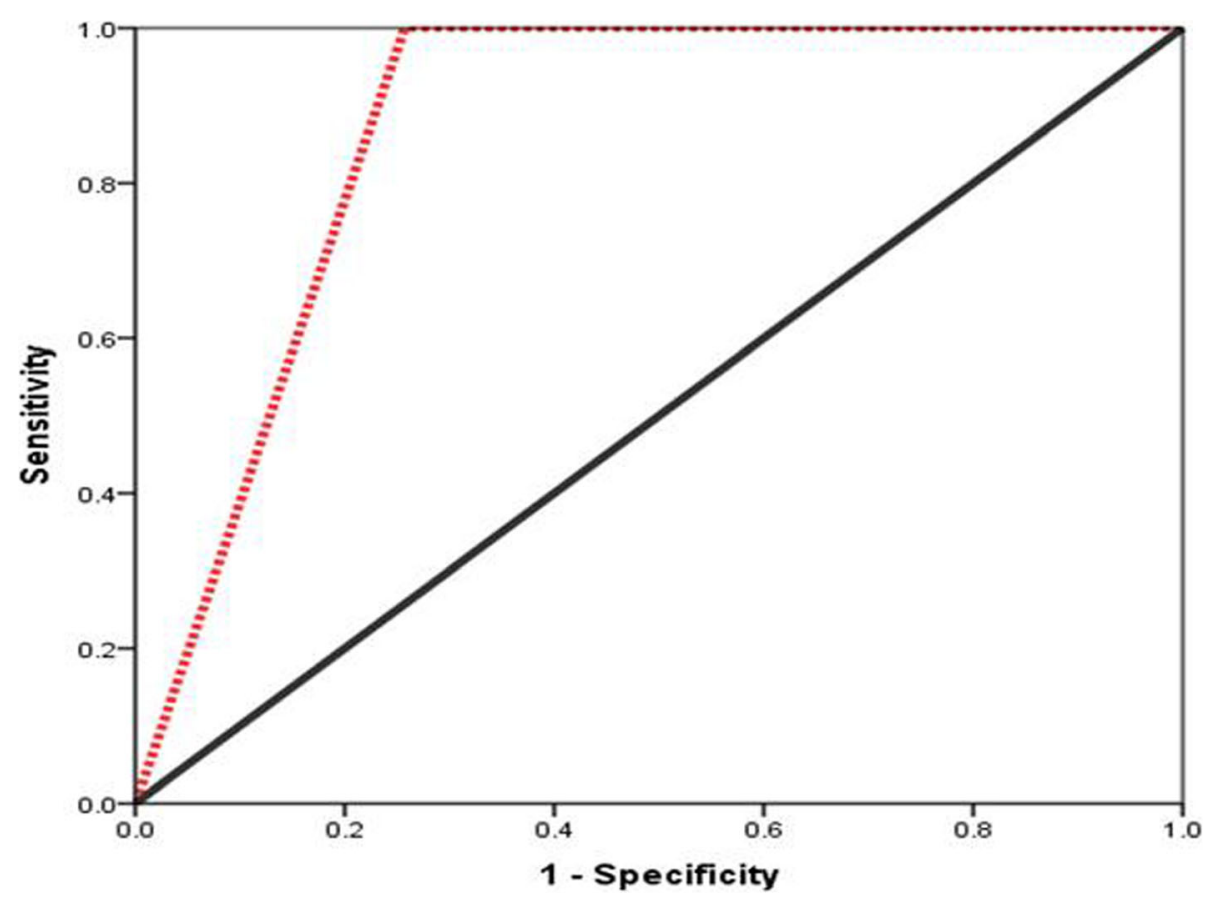

Fig. 1 ROC curve for malignancy prediction via DCE-MRI 


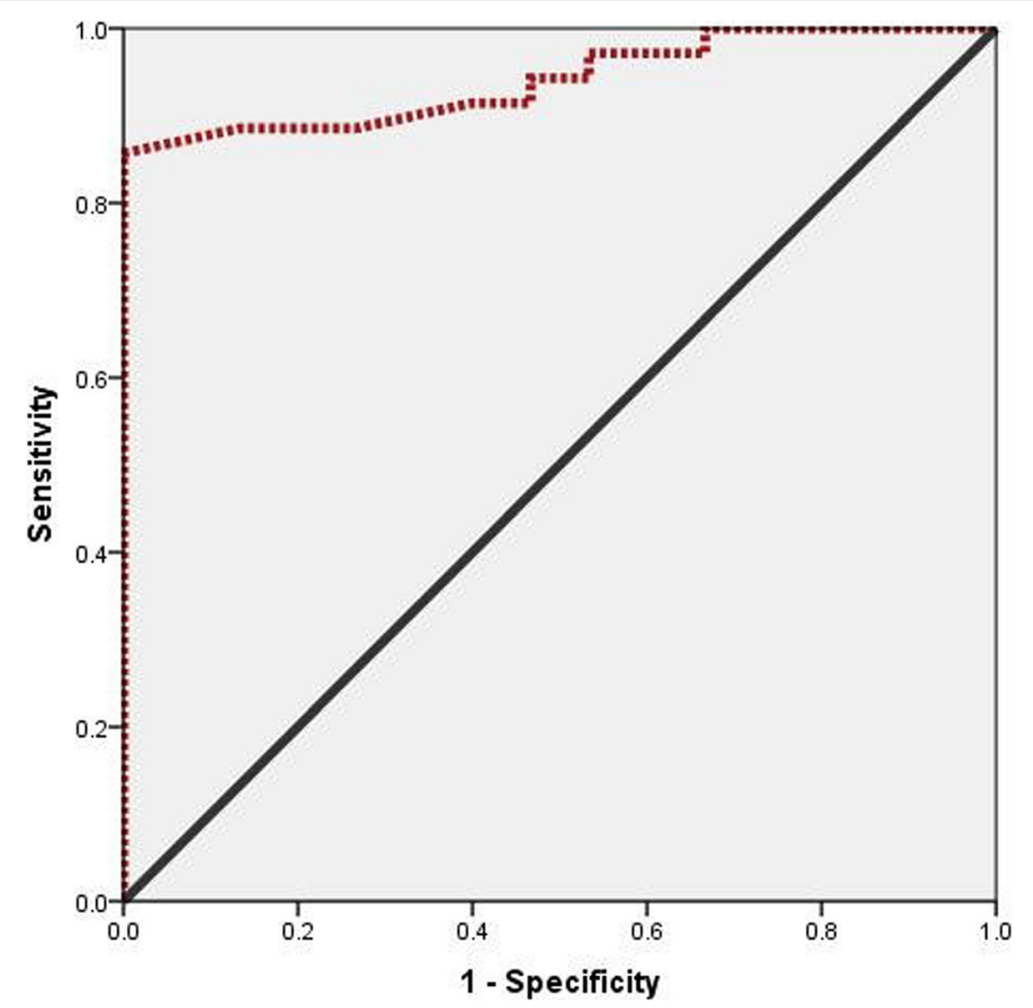

Fig. 2 ROC curve analysis for the ADC value

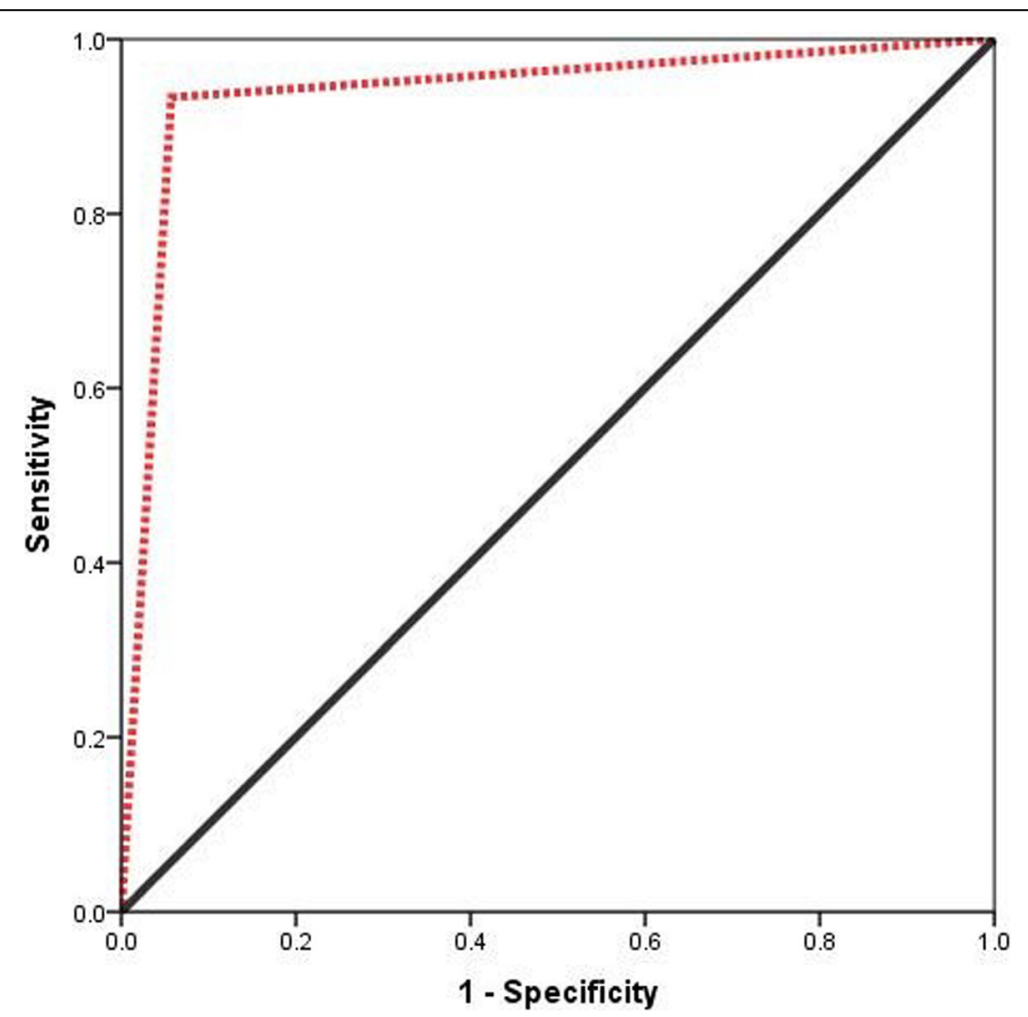

Fig. 3 ROC curve for malignancy prediction via the combined protocol of DCE-MRI and DWI 
almost benign [5]. Similarly, Badawi, and Amin showed in their study on 86 female patients with asymmetric density that $72 \%$ of cases were attributed to benign mammary changes [12] (Figs. 4, 5, 6, 7, and 8).

In the present study, there was no statistically significant difference between malignant and benign lesions according to the type of asymmetry. However, the likelihood of malignant etiology was higher with focal asymmetry and developing asymmetry than with one view and global asymmetry. Youk et al. reported that the likelihood of cancer varies depending on the type of asymmetry [6]. Sickles reported that the likelihood of malignancy for one view asymmetry, non-palpable and palpable global asymmetry, focal asymmetry, and developing asymmetry were $1.8 \%$, $0 \%, 7.5 \%, 0.67 \%$, and $12.8 \%$, respectively [5].

In our study, the final mammographic assessment was achieved after evaluation of the presence or absence of other associated suspicious findings as suspicious calcification, skin and nipple changes, and suspicious lymph nodes, and there was a statistically significant difference between benign and malignant lesions according to the final mammographic BIRADS assessment. This is in concordance with Wessam et al. who concluded from their study that focal and global asymmetries with other suspicious mammographic findings were statistically significant for malignancy, and there was a significant correlation between asymmetry associated with distortion, suspicious calcification, skin/nipple changes, and malignancy. Focal and global asymmetries with no other associated mammographic findings were significantly correlated with a benign pathology [13]. Similarly, Harvey et al. considered focal asymmetry as being more suspicious than global asymmetry, especially if companion parenchymal distortion is present [14].
In our study, the histopathological diagnosis of 35 benign lesions revealed that the most frequent lesions were focal fibrocystic disease 12 (34.2\%), inflammatory changes 9 (25.7\%) (chronic mastitis, periductal mastitis and inflammatory changes with abscess formation), and postoperative sequelae 5 (14.2\%) either postoperative seroma, organized hematoma, or fibrotic surgical scar (postoperative granulation tissue). This agrees with Badawi and Amin's and Moy et al.'s studies which showed that fibrocystic changes represented the most common cause of mammographic asymmetric breast densities $[12,15]$. Wessam et al.'s study showed that inflammatory causes (granulomatous mastitis and breast abscess) were the most common benign etiology of asymmetric densities [13].

The pathologic results of 15 malignant lesions revealed that the most common malignant lesion was invasive duct carcinoma 7 (46.6\%). This agrees with Moy et al.'s study which showed that invasive ductal carcinoma being the most common malignant cause of asymmetric densities [15] and in concordance with Wessam et al.'s study which showed that invasive ductal carcinoma and invasive lobular carcinoma were the most common malignant etiologies of asymmetric densities. In our study, only one case $(6.6 \%)$ was invasive lobular carcinoma [13]. In a study done by Brenner, he found that infiltrating lobular carcinoma lacks adhesive substances on the cell membrane and often invade the breast as cords of cells without creating a recognizable mass. So, he considered that a focal asymmetric density or density that is increasing in size is often associated with the diagnosis of infiltrating lobular carcinoma [16].

The calculated sensitivity and specificity of mammography in the characterization of benign and malignant
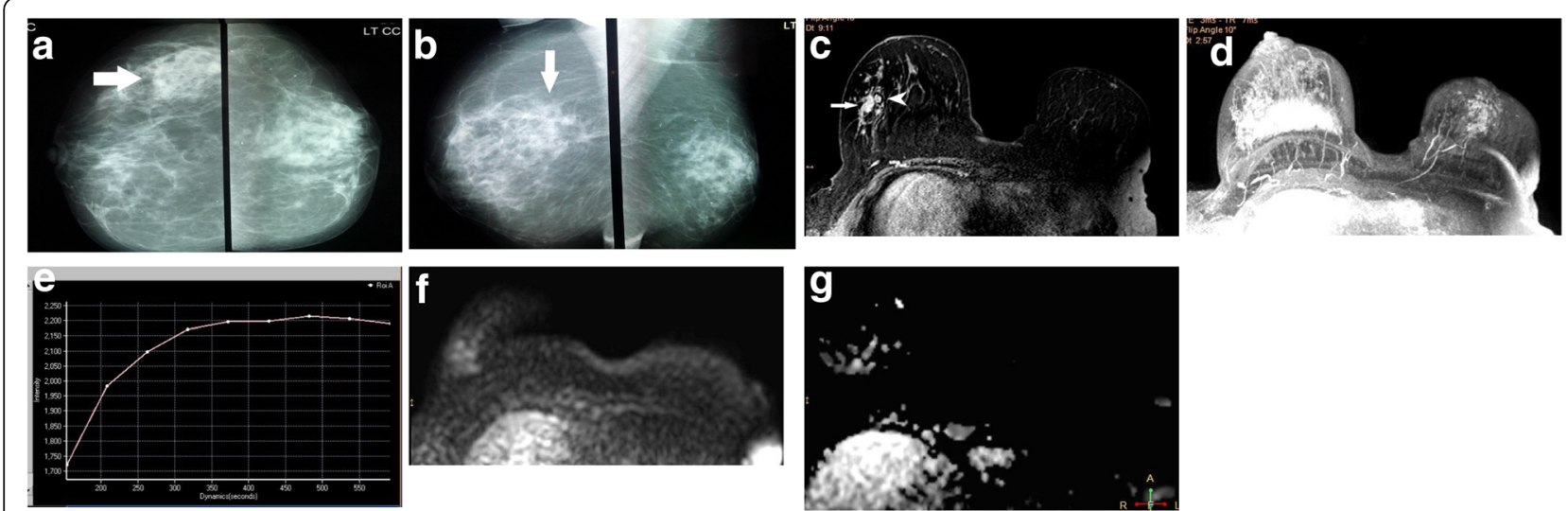

Fig. 4 A 45-year-old female patient with right breast lump. Mammography craniocaudal (a) and mediolateral oblique (b) views show focal asymmetry (arrow) in the right upper outer quadrant (BIRADS IV lesion). Axial TI contrast-enhanced fat-suppressed image on dynamic sequence (c) showed homogenous segmental non-mass enhancement (NME) (arrow) with well-defined enhanced intraductal lesion (arrow head). Axial MIP image (d) shows the right breast non-mass enhancement with increased vascularity to right breast. Dynamic time-intensity curve (e) shows type II "plateau" curve. The lesion elicits faint high signal intensity on DWI at $b$ value $=400$ ( $\mathbf{f}$ ) with a mean $\mathrm{ADC}$ value of $1.2 \times 10^{-3} \mathrm{~cm} / \mathrm{s}$ on $\mathrm{ADC}$ map (g). MRI findings: BIRADS IV lesion. Histopathological diagnosis: atypical ductal hyperplasia 


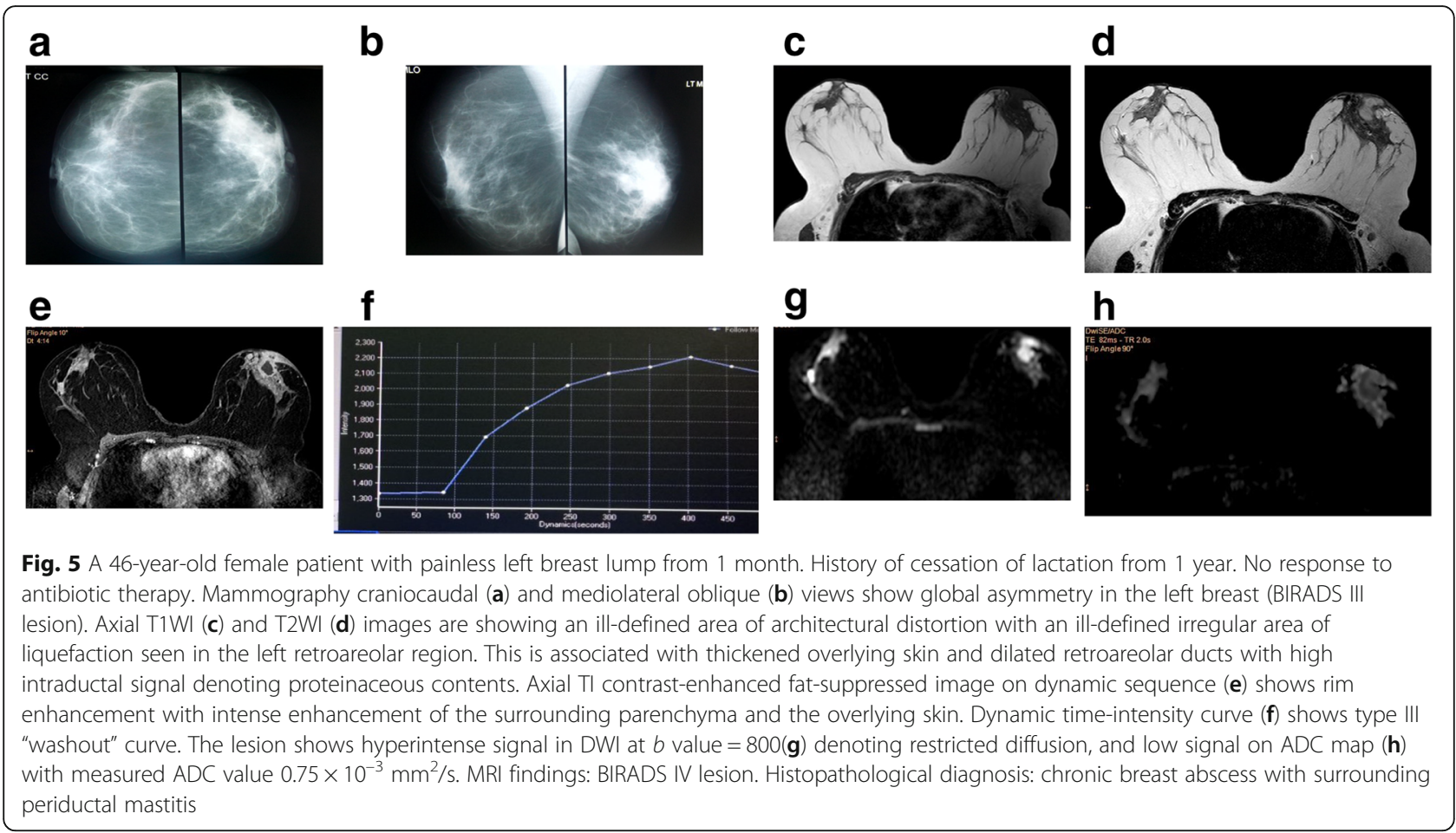

asymmetries were $47 \%$ and $91.5 \%$, respectively. The positive predictive value was $70 \%$, negative predictive value $80 \%$, and accuracy $78 \%$. Our results were nearly the same as Moy et al. who reported sensitivity $33.3 \%$, specificity $80.7 \%$, and accuracy $78.3 \%$ [15]. On the other hand, Wessam et al. reported higher sensitivity being $97.8 \%$, with specificity $81.8 \%$, PPV 93.7\%, and NPV 93\% [13].
MRI examination showed that from 50 lesions, 25 (50\%) masses were found (16 "64\%" were benign and 9 "36\%" malignant) and 25 (50\%) were non-mass-like enhancement (19 "76\%" were benign and 6 "24\%" malignant). There was no significant correlation between the type of enhancement and malignant pathology. Wessam et al. reported that any enhancing asymmetry with mass
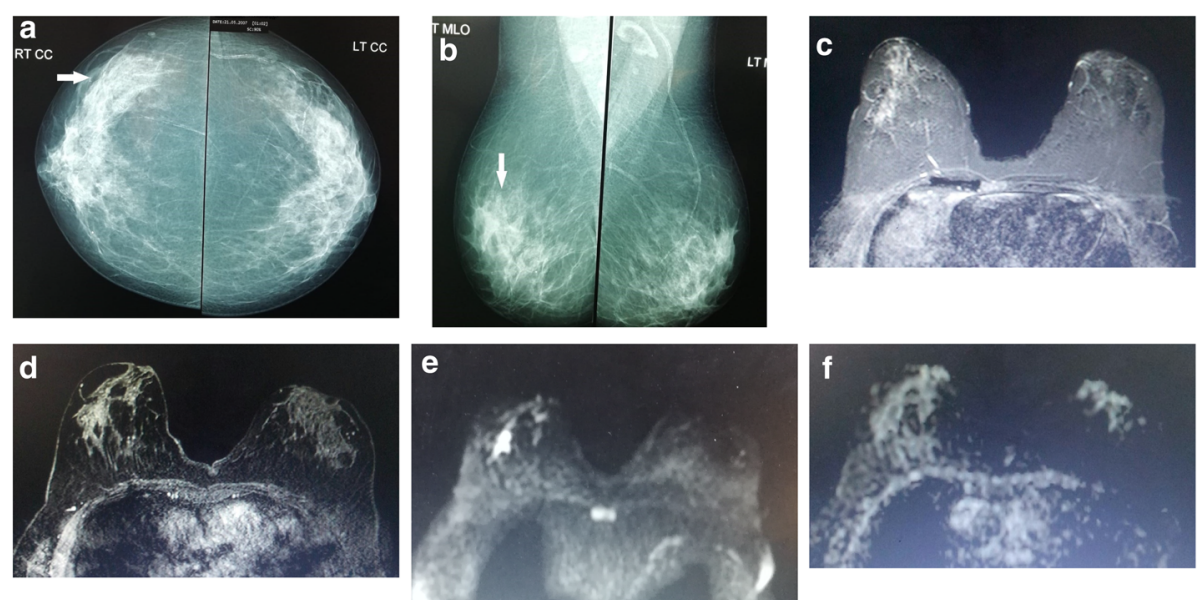

Fig. 6 A 68-year-old female patient with right mastalgia. Mammography craniocaudal (a) and mediolateral oblique (b) views show focal asymmetry in the right upper quadrant. This is associated with suspicious amorphous microcalcifications (BIRADS IV lesion). Axial STIR (c) image shows an ill-defined hyperintense segmental area seen in the right upper outer quadrant with dilated ducts. Axial TI contrast-enhanced fatsuppressed image on dynamic sequence (d) shows heterogeneous segmental non-mass enhancement with linear ductal extension towards the nipple. Dynamic time-intensity curve shows type II plateau curve (not shown). The lesion shows hyperintense signal in DWI at $b$ value $=400$ (e) denoting restricted diffusion, and low signal on ADC map ( $\mathbf{f}$ ) with measured ADC value $1.22 \times 10^{-3} \mathrm{~mm}^{2} / \mathrm{s}$. MRI findings: BIRADS IV lesion. Histopathological diagnosis: usual ductal hyperplasia 


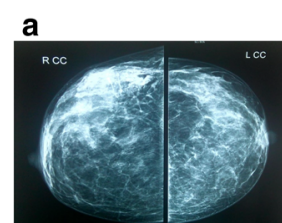

e

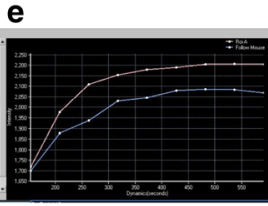

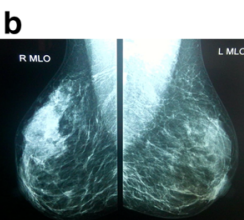

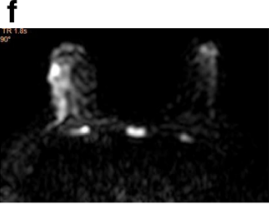

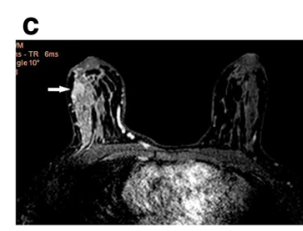

g

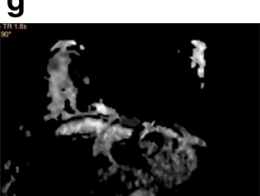

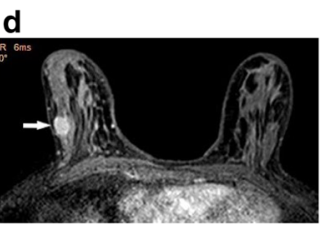

h

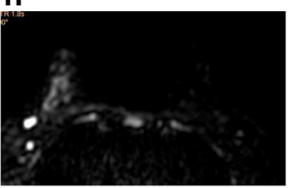

Fig. 7 A 42-year-old female patient with breast lump in the upper outer quadrant of the right breast. Mammography craniocaudal (a) and mediolateral obliquel (b) views show focal asymmetry in the right upper outer quadrant, associated with pleomorphic microcalcifications (BIRADS IV lesion). Axial TI contrast-enhanced fat-suppressed image on dynamic sequence shows an intensely enhancing irregular right breast mass (arrow) surrounded by segmental area of increased parynchemal enhancement (c). Another smaller enhanced well-defined regular mass (D) seen posteroinferiorly (arrow) to the previous mass, suggesting multicentric carcinoma. Dynamic time-intensity curve (e) shows type II "plateau" curve. The lesion shows hyperintense signal in DWI (f) denoting restricted diffusion, and low signal on ADC map (g) with measured ADC value $0.56 \times$ $10-3 \mathrm{~mm}^{2} / \mathrm{s}$. Restricted diffusion of the ipsilateral axillary lymph nodes (h) with low ADC value $0.67 \times 10-3 \mathrm{~mm}^{2} / \mathrm{s}$. MRI findings: BIRADS V lesion. Histopathological diagnosis: Invasive ductal carcinoma

or non-mass enhancement was significantly correlated with malignant pathology [13].

Regarding kinetic curve assessment, type III was significantly correlated to malignant pathology for the pathologically proved 15 malignant lesions ( 9 of them showed type III curve, and 6 showed type II curve). On the other hand, type I curve was significantly correlated with benign pathology. There was statistically significant difference between benign and malignant according to type of curve. Tozaki and Fukuda's and Yabuuchi et al.'s studies on 45 NME lesions revealed no significant difference in kinetic curve assessment between benign and malignant lesions, yet they attributed this to the dual tendency of non-invasive ductal carcinoma to show both persistent and plateau/washout patterns $[17,18]$.

This study showed that dynamic contrast-enhanced MRI had sensitivity and specificity in the characterization of benign and malignant lesions of $100 \%$ and $74.3 \%$, respectively. The positive predictive value was $62.5 \%$, negative predictive value was $100 \%$, and accuracy was $82 \%$. Moy et al. had emphasized on the role of MRI as a problem solver in cases of inconclusive mammographic

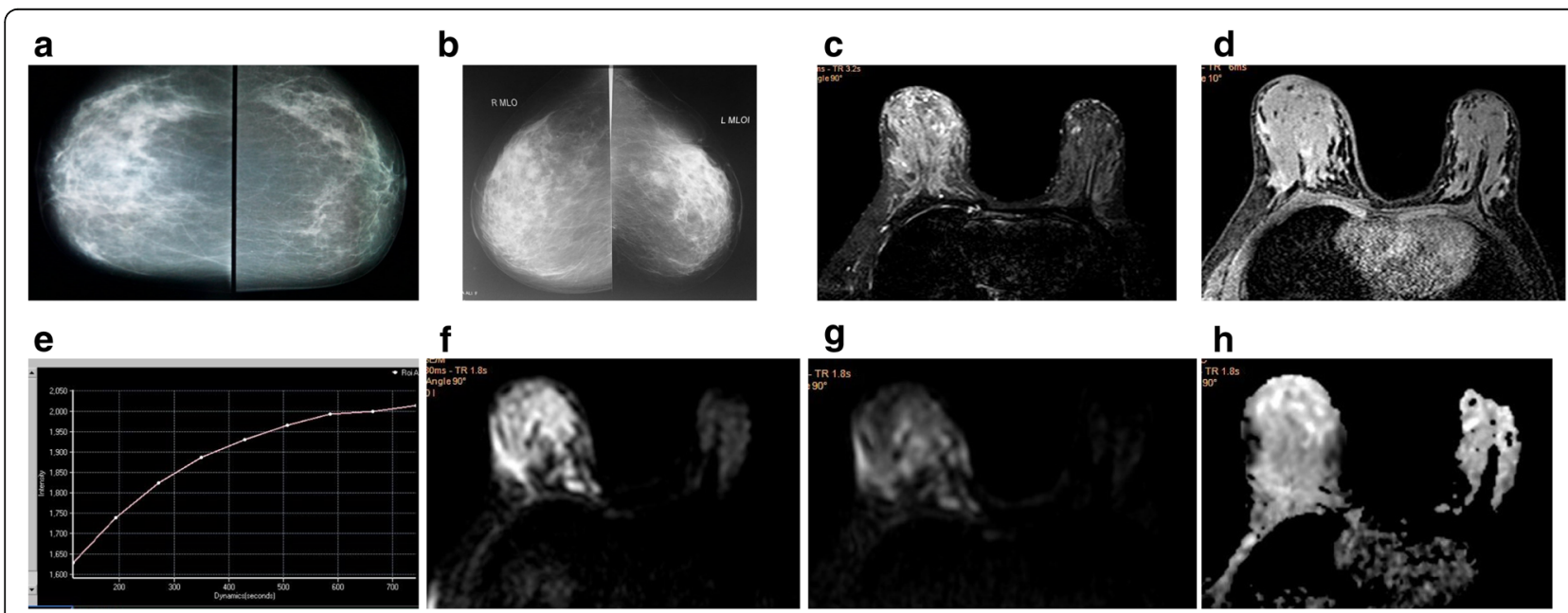

Fig. 8 A 37-year-old female patient with right mastalgia and breast tenderness for 3 weeks, not relieved with antibiotic therapy. Mammography craniocaudal (a) and mediolateral oblique (b) views show global asymmetry in the right breast (BIRADS II lesion). Axial STIR (c) is showing diffuse increased the signal intensity of the right breast parenchyma. Axial TI contrast-enhanced fat-suppressed image on dynamic sequence (d) shows diffuse homogenous non-mass enhancement of the right breast. Dynamic time-intensity curve (e) shows type I "progressive enhancement" curve. The lesion shows high signal on DWI at $b$ value $=200(\mathbf{f})$ and a very faint hyper intense signal at $b$ value $=800(\mathbf{g})$ with $\mathrm{ADC}$ value $1.68 \times 10$ $3 \mathrm{~mm}^{2} / \mathrm{s}$ (h). MRI findings: BIRADS III lesion. Histopathological diagnosis: chronic mastitis 
findings including breast asymmetries. They reported sensitivity of $100 \%$, specificity $91.7 \%$, negative predictive value $100 \%$, positive predictive value $40 \%$, and accuracy $92.2 \%$ [15].

The mean ADC value for benign lesions was $1.59 \pm$ $0.4 \times 10^{-3} \mathrm{~mm}^{2} / \mathrm{s}$, and for malignant lesions, it was $0.82 \pm 0.3 \times 10^{-3} \mathrm{~mm}^{2} / \mathrm{s}$. For benign lesions, the ADC value was ranging from $0.63 \times 10^{-} 3 \mathrm{~mm}^{2} / \mathrm{s}$ to $2.01 \times$ $10^{-3} \mathrm{~mm}^{2} / \mathrm{s}$, and for malignant lesions, it was ranging from $0.38 \times 10^{-3} \mathrm{~mm}^{2} / \mathrm{s}$ to $2.21 \times 10^{-3} \mathrm{~mm}^{2} / \mathrm{s}$. Abd ElAleem et al. reported a nearly similar ADC values. The mean ADC value for benign lesions was $1.54 \pm 0.43 \times$ $10^{-3} \mathrm{~mm}^{2} / \mathrm{s}$. The mean ADC value for malignant lesions was $0.93 \pm 0.42 \times 10^{-3} \mathrm{~mm}^{2} / \mathrm{s}$. The range of ADC values for malignant lesions was $0.51-1.35$, and for benign lesions was $0.86-1.97$ [19].

This study showed that the best ADC cutoff value to differentiate between benign and malignant lesions was $1.10 \times 10^{-3} \mathrm{~mm}^{2} / \mathrm{s}$ with sensitivity $80 \%$, specificity $88.6 \%$, positive predictive value $75 \%$, negative predictive value $91 \%$, and accuracy $86 \%$. Abd El-Aleem et al. reported an ADC value of $1.26 \times 10^{-3} \mathrm{~mm}^{2} / \mathrm{s}$ as a cutoff value to differentiate between benign and malignant lesions with sensitivity and specificity of $89 \%$ and $94.7 \%$, respectively [19]. Similarly, Yabuuchi et al. reported the ADC value of less than $1.3 \times 10^{-3} \mathrm{~mm}^{2} / \mathrm{s}$ was a significant factor indicating malignancy among the non-mass enhancement lesions [18].

The majority of benign lesions 31/35 (88.6\%) had the ADC value above the calculated cutoff value of $1.1 \times 10^{-3} \mathrm{~mm}^{2} / \mathrm{s}$, while only 4/35 (11.4\%) had ADC value below the cutoff value (the lesions were chronic breast abscesses). This is in agreement with Rubesova et al. who reported that inflammatory changes can show low ADC values, probably due to high cellularity, fibrosis, and leukocytes [10]. Similarly, Abd El-Aleem et al. reported one false positive with low ADC value, and it was mastitis [19].

In our study, the majority of malignant lesions $12 / 15$ $(80 \%)$ had the ADC value below the calculated cutoff value of $1.1 \times 10^{-3} \mathrm{~mm}^{2} / \mathrm{s}$, while only $3 / 15(20 \%)$ had the $\mathrm{ADC}$ value above the cutoff value (the lesions were atypical ductal hyperplasia and ductal carcinoma in situ). Woodhams et al. illustrated that mass-forming DCIS will show high signal intensity in DWI due to its relatively high cellularity. However, the signal intensity of lowgrade DCIS with low cellularity may be ambiguous. Moreover, DWI has a low spatial resolution, and thus, small cancer foci such as DCIS may not be depicted. DCIS and invasive lobular carcinoma may contain interspersed normal fibroglandular tissue or fat tissue, which can increase the ADC value [20].

Lima et al. reported false negative cases comprised a small IDC and a DCIS presenting as NME with borderline ADC values [21]. Similarly, Guo et al. reported that DCIS and malignant phyllodes tumor with bleeding show high ADC values as a result of the strong effects of magnetic susceptibility. Malignant phyllodes tumor can have high ADC values due to cystic areas inside the tumor [22].

Yabuuchi et al. illustrated that non-mass enhancement lesions can form large non-compact lesions, with normal parenchyma in the center of the tumor. Thus, a lesser diffusion restriction occurs. This has been reported for several pathologic and normal states, including DCIS, LCIS, atypical ductal hyperplasia, papillomas, hormonal changes, and fibrocystic disease [18].

Woodhams et al. reported one false negative result with a high ADC value was a mucinous carcinoma, owing to its mucinous content and low cellularity [20]. However, in our study, the depicted case of mucinous carcinoma had an ADC value of $0.56 \times 10^{-3} \mathrm{~mm}^{2} / \mathrm{s}$.

The best specificity and accuracy measures were achieved by the use of the combined protocol of DCEMRI and DWI. The calculated sensitivity and specificity were $93.3 \%$ and $94.3 \%$, respectively. The positive predictive value was $87.5 \%$, negative predictive value $97.1 \%$, and accuracy $94 \%$. DWI was a useful adjunctive measure that maintained the high sensitivity, increased specificity, and maximized the overall diagnostic accuracy of the breast MRI examination. Yabuuchi et al. reported that the combination of morphology and ADC values had a high prediction probability for malignancy and showed the high accuracy [18].

\section{Conclusion}

Breast asymmetry is a perplexing finding that is caused by a spectrum of normal variations and different pathologies. Benign pathology is the most frequent underlying pathology for breast asymmetries. Mammography allows good detection, localization, and preliminary BIRADS classification of the underlying pathology of breast asymmetry. However, it has a limited sensitivity and specificity for cancer detection.

DCE-MRI is the most sensitive method for the detection of the underlying malignant pathology of breast asymmetries. However, it provides a limited specificity that may cause improper final BIRADS classification and increases the unnecessary invasive procedures.

Combination of DCE-MRI with a functional imaging procedure as DWI provides both qualitative and quantitative assessment and allows more characterization of breast lesions, thus permitting a more confident management. DWI is a useful adjunctive measure that maintained the high sensitivity, increased specificity, and the overall diagnostic accuracy of breast MRI examination. The best specificity and accuracy can be achieved by the combined protocol of DCE-MRI and DWI. 


\section{Recommendation}

We recommended further studies with a larger sample size on the role of DWI in the assessment of asymmetric findings frequently detected on screening programs. DWI can be ideal for this purpose, because it is a rapid sequence that can be used safely with pregnant females and patients in whom contrast administration is contraindicated. However, DWI has a low spatial resolution, and anatomical and morphological guidance with other non-enhanced MRI sequence could overcome this limitation. We think that an abbreviated protocol (T1WI, T2WI, STIR, DWI, and ADC map) might be suitable for screening that allows a more confident decision of short-term follow-up for cases of one view asymmetry and other asymmetric densities attributed to normal hormonal variations, hormonal replacement therapy, focal fibroadenosis, or subtle postoperative changes.

\section{Abbreviations}

ACR: American College of Radiology; ADC: Apparent diffusion coefficient; AUC: Area under the curve; BI-RADS:: Breast imaging and reporting data system; CC: Cranio-caudal; DCE-MRI: Dynamic contrast-enhanced magnetic resonance imaging; DCIS: Ductal carcinoma in situ; DWI: Diffusion-weighted imaging; EPI: Echo-planer imaging; MIP: Maximum intensity projection; MLO: Medio-lateral oblique; NME: Non-mass enhancement; PPM: Parts per million; ROC: Receiver operating characteristic curve; ROI: Region of interest

\section{Acknowledgments}

The authors thank all the study participants for their patience and support.

\section{Authors' contributions}

E. A. A. suggested and developed the research idea and reviewed the literature. E. A. A., H. E. G., M. Z. M., R. E., and S. A. S. were responsible for data collection and analysis, performed statistical analysis, wrote and revised the manuscript, prepared cases, performed the required measurements, and prepared the figures and tables. E. A. A. and S. A. S. were responsible for reporting the cases of mammography and diffusion-weighted MRI and compared it with histopathological results. All authors have a major contribution in preparing and editing the manuscript. All authors read and approved the final manuscript.

\section{Funding}

No funding was obtained for this study.

\section{Availability of data and materials \\ The data that support the findings of this study are available from the Radiology Department, Assiut University, but there are restrictions applied to the availability of data, which was used under license for this study, and so were not publicly available. Data were available from the authors upon request with permission of the head of the Radiology Department, Assiut University.}

\section{Ethics approval and consent to participate}

This study had approval from Egypt, Assiut University, Faculty of Medicine Research Ethics Committee, 71515, Tel: +208822080150 . All patients who participated in this study signed an informed written consent for participation.

\section{Consent for publication}

All patients included in this research gave written informed consent to publish the data contained within this study.

\section{Competing interests}

The authors declared that they had no competing interests.

\section{Author details}

${ }^{1}$ Department of Radiology, Assiut University, Asyut, Egypt. ${ }^{2}$ Department of Radiology, Taibah University, Madina, Saudi Arabia.
Received: 28 July 2020 Accepted: 13 November 2020

Published online: 02 December 2020

\section{References}

1. Kopans DB, Swann CA, White G, McCarthy KA, Hall DA, Belmonte SJ, Gallagher W (1989) Asymmetric breast tissue. Radiology 1989(171):639-643

2. Samardar P, Shaw de Paredes E, Grimes MM, Wilson JD (2002) Focal asymmetric densities seen at mammography: US and pathologic correlation. Radio-Graphics 2002(22):19-33

3. Berg WA, Campassi C, Langenberg P, Sexton MJ (2000) Breast Imaging Reporting and Data System: inter and intraobserver variability in feature analysis and final assessment. Am J Roentgenol 2000(174):1769-1777

4. American College of Radiology (2013) Breast Imaging Reporting and Data System ${ }^{\circ}$ (BI-RADS ${ }^{\circ}$ ), 5th edn. American College of Radiology, Reston

5. Sickles EA. The spectrum of breast asymmetries: imaging features, work-up, and management. Radiol Clin North Am 2007;45:765-771, v.

6. Youk JH, Kim EK, Ko KH, Kim MJ. Asymmetric mammographic findings based on the fourth edition of BI-RADS: types, evaluation, and management. RadioGraphics 2009 29:1 e33-e33.

7. Nunes W, Schnall MD, Orel SG. Update of breast MR imaging architectural interpretation model. Radiology 2001;219(2): 484-94.

8. Yau EJ, Gutierrez RL, DeMartini WB et al (2011) The utility of breast MRI as a problem-solving tool. Breast J 2011(17):273-280

9. Wenkel E, Geppert C, Schulz-Wendtland R, Uder M, Kiefer B, Bautz W, Janka $R$ (2007) Diffusion weighted imaging in breast MRI: comparison of two different pulse sequences. AcadRadiol 14(9):1077-1083

10. Rubesova E, Grell AS, De Maertelaer V, Metens T, Chao SL, Lemort M (2006) Quantitative diffusion imaging in breast cancer: a clinical prospective study. J MagnReson Imaging 2006(24):319-324

11. Thomassin-Naggara I, Trop I, Lalonde L, David J, Péloquin L, Chopier J. Tips and techniques in breast MRI. Diagnostic Interventional Imaging.2012, 93(11):828-839.

12. Badawi HA, Amin AA (2010) The role of MRI in assessment of asymmetrical breast densities. Egyptian J Radiol Nuclear Med 41:501-508

13. Wessam R, Gomaa MM, Fouad MA, Mokhtar SM, Tohamey YM (2019) Added value of contrast-enhanced mammography in assessment of breast asymmetries. Br J Radiol 2019(92):20180245

14. Harvey JA, Nicholson BT, Cohen MA (2008) Finding early invasive breast cancers. Radiology 2008(248):61-76

15. Moy L, Elias K, Patel V et al (2009) Is breast MRI helpful in the evaluation of inconclusive mammographic findings? Am J Roentgenol 2009(193):986-993

16. Brenner RJ. Asymmetric densities of the breast: strategies for imaging evaluation. seminars in Roentgenology, Vol XXXVI, No 3, 2001: pp 201-216.

17. Tozaki M, Fukuda K. High-spatial-resolution MRI of non-mass like breast lesions: interpretation model based on BI-RADS MRI descriptors. AJR Am J Roentgenol 2006;187(2):330-7.

18. Yabuuchi H, Matsuo Y, Kamitani T, Setoguchi T, Okafuji T, Soeda H, Sakai S, Hatakenaka M, Kubo M, Tokunaga E, Yamamoto H, Honda H (2010) Nonmass-like enhancement on contrast-enhanced breast MR imaging: Lesion characterization using combination of dynamic contrast-enhanced and diffusion-weighted MR images. Eur J Radiol 75:e126-e132

19. Abd El-Aleem RA, Abo El-Hamd E, Yousef HA, Radwana MEM, Mohammed RA (2018) The added value of qualitative and quantitative diffusionweighted magnetic resonance imaging (DW-MRI) in differentiating benign from malignant breast lesions. Egyptian J Radiol Nuclear Med 49:272-280

20. Woodhams R, Kakita S, Hata H et al (2009) Diffusion-weighted imaging of mucinous carcinoma of the breast: evaluation of apparent diffusion coefficient and signal intensity in correlation with histologic findings. AJR Am J Roentgenol 2009(193):260-266

21. Lima M, Le Bihan D, Okumura R et al (2011) Apparent diffusion coefficient as an MR imaging biomarker of low-risk ductal carcinoma in situ: a pilot study. Radiology 2011(260):364-372

22. Guo Y, Cai YQ, Cai ZL, Gao YG, An NY, Ma L, Mahankali S, Gao JH (2002) Differentiation of clinically benign and malignant breast lesions using diffusion-weighted imaging. J Magnetic Resonance Imaging 16(2):172-8

\section{Publisher's Note}

Springer Nature remains neutral with regard to jurisdictional claims in published maps and institutional affiliations. 